\title{
A multi-faceted approach to increase appropriate analgesia prescribing in the emergency department
}

\author{
Culadeeban Ratneswaran, Kevin Dodd, Kevin Enright, Sunil Dasan \\ St George's University Hospitals NHS Foundation Trust, United Kingdom
}

\begin{abstract}
Pain is the most common presenting complaint within the emergency department. Whilst national RCEM guidelines exist, there tends to be low compliance with its use. A retrospective, cross-sectional audit, over a 24 hour period, was carried out in the emergency department of a tertiary hospital in London on all patients with abdominal pain. Pain score documentation was checked as well as: whether analgesia prescribed was compliant with guidelines, time to prescription, and if pain scores were rechecked within an hour.
\end{abstract}

Cycle 1 (21 patients) showed that only $29 \%$ of patients were prescribed analgesia in accordance with guidelines, $38 \%$ of pain scores were documented at triage, and only $19 \%$ of scores were rechecked at any time. $22 \%$ of patients in severe pain were prescribed analgesia within the recommended duration from presentation (20 minutes).

New guidelines, adapted from RCEM, were departmentally approved and disseminated to reflect local medication use. Monthly doctor and nurse teaching sessions were established to improve guideline compliance, objective pain score documentation, and encourage results driven performance. A nurse prescriber champion was established to encourage analgesia prescribing competence in addressing delayed administration. Finally, plans to integrate electronic pain scoring with timer prompts for rechecking are in place to help streamline the process.

Following these interventions, cycle $2(n=23)$ showed $87 \%$ of pain scores were documented at triage, $52 \%$ were prescribed guideline concordant analgesia, and $40 \%$ of severe pain scores were acted upon in time. Cycle $3(n=33)$ demonstrated the need for monthly educational intervention to maintain high standards; as in its absence, any improvement returned to baseline.

\section{Problem}

Whilst the Royal College of Emergency Medicine (RCEM) national guideline exists,[1] our emergency department service improvement team identified a discrepancy between this and locally stocked medications. Furthermore, there was a low awareness of the national guideline and low adequate pain scoring. This is likely to be the case in other emergency departments (ED) throughout the country, decreasing compliance with recommended algorithms. In effect, patients presenting to the ED would remain in more pain and for longer.

\section{Background}

Pain is the most common presenting complaint within the emergency department (ED) with up to $75 \%$ of patients presenting with pain as their primary symptom.[2,3] Pain relief on the other hand, within this setting is poor.[4] This has a significant effect on patients' perception of care leading to a number of consequences including a reduction in patient rapport and a decreased compliance with medical advice.[5] Poorer perception of care may lead to an increased number of complaints with decreased satisfaction in service provision, as shown in one Australian Emergency department, where up to $33.4 \%$ of complaints were related to patient treatment.[6]
Severe pain may act as a barrier between the patient and physician, leading to inadequate history taking and clinical examination.[7] From a managerial perspective, it has been shown that administration of analgesia within even 90 minutes, can reduce ED length of stay by two hours.[8]

Another barrier to effective pain relief includes inadequate scoring of pain. Several studies indicate that a discrepancy exists between patients' perceived pain score and the subjective evaluation of their pain by medical professionals, with nurses showing the greatest disparity $[4,9]$. This has importance as nurses are usually the first team members to initiate a patient's management plan in the ED.

Whilst initial pain scoring and prescription is important, staff must ensure that this is then also administered. Todd et al.[3] showed an average time to administration of analgesia was 90 minutes with only $29 \%$ of patients receiving analgesia within an hour.

Furthermore, there is often no re-evaluation of a patient's pain after receiving initial analgesia. In one UK paediatric emergency department 0 of the 100 patients audited had their pain reassessed.[10] A lack of communication between staff may be a contributory factor here; however, this may also be due to simple inadequate documentation of the consultation.

\section{Baseline measurement}

Cycle 1 (21 patients) showed that only $38 \%$ of pain scores were 
documented at triage. This meant that the majority of patients had unknown pain scores by the time they had entered the main ED, delaying appropriate prescribing and within the correct time. Only $29 \%$ of patients were prescribed analgesia in accordance with guidelines and only $19 \%$ of pain scores were documented as having been rechecked at any time. $22 \%$ of patients in severe pain were given or prescribed analgesia within the recommended duration from presentation (20 minutes, online supplement [OS] table 1).

See supplementary file: ds6141.pdf - "Online supplement table 1"

\section{Design}

This was a retrospective cross-sectional audit performed on all patients presenting with abdominal pain over a 24 hour period, with monthly reauditing. A coded excel spread sheet calculated our outcome measures i.e. analgesia compliance with the algorithm, time until prescription, and time of reassessment based on the following inputted values: time of ED presentation, time of prescription, time to discharge, pain score documentation, analgesia prescribed (if offered), and documentation of rechecking.

\section{Strategy}

PDSA cycle 1:

Following baseline measurements, a number of barriers became apparent. There was a low compliance with guidelines and this was multifactorial. Firstly knowledge that national ED pain guidelines existed was low; secondly, RCEM guidelines did not include some locally used medications; and thirdly, nurses were not documenting objective pain scores on patients' presentation at triage. There was a three pronged approach in addressing this: a) creation of new guidelines, b) guideline dissemination, and c) doctor and nursing educational sessions.

Cycle 1 outcome A - Adaptation of national RCEM guidelines and dissemination

RCEM guidelines did not include some commonly used local medications; these were codydramol, dihydrocodeine, and subcutaneously administered morphine. Though easily integrated, discrepancies may discourage guideline use, especially amongst new or junior staff with less confidence in doing so.

An adaptation of the RCEM algorithm was created with these medications included in the relevant area (OS figure 1). Following departmental consultant approval, this was made available on the hospital intranet and posters were placed in key staffing areas: lunch room, seminar doorways, and staff entrance to the emergency departments in order to allow for maximal staff exposure. These were visually appealing to draw attention to, and consolidate the guidelines.

[Online supplement figure 1]
Cycle 1 outcome B - Education of doctor and nursing teams

Once monthly, before any repeat audit, a 10 minute education session was carried out in the foundation year and registrar teaching sessions. This consisted of emphasising the consequences of poor analgesia prescribing i.e. decreased patient satisfaction, and presenting the previous audit cycle results to encourage results driven performance. Leaflets of the adapted pain relief algorithm were also distributed here.

Nursing teaching sessions took place monthly during handovers, with a greater emphasis on the need to seek analgesia early. Both groups were encouraged to not only document the patients' subjective pain score, but also their own objective assessment. This would avoid the prescribed analgesia being misinterpreted by our audit as poorly compliant with guidelines.

Cycle 1 outcome C - Nurse prescriber sign-offs

None of the nurses on our initial audit day had prescriber books signed. This led to delayed analgesia administration because of a lack of prescribing ability. This perhaps also fostered a decreased perceived necessity to document pain scores, as the nurses would have been unable to act upon them. A lead nurse was allocated to encourage prescriber sign offs.

PDSA cycle 2:

Significant improvements were noted in pain scoring and guideline compliance after the given interventions, however documentation of rechecked pain scores remained low at $22 \%$. Identified barriers include a high cognitive demand in the ED in addition to managing multiple patients.

Cycle 2 outcome - Integrated clinical information system: electronic pain scoring

Following these results, plans are in place to implement an electronic pain score and rechecking timer prompt on the EDs integrated clinical information system (Cerner Millennium®). This is visible on all ED computers and regularly referred to for patient updates, so it should streamline the process to rechecking, as well as addressing issues of poor documentation.

PDSA cycle 3 and outcome - sustainability and continuous cycle of auditing

A drop in standards back to baseline was noted when there was no educational intervention between cycles 2 and 3 (OS figure 2). A junior doctor from each four monthly cohort was recruited in an ongoing continuous cycle of monthly auditing and educational intervention to maintain standards. Carrying out an audit process is a requirement to pass foundation year training, hence junior doctors are incentivised to help.

PDSA cycle 4 and outcome - confirming a rise in standards and the need for an electronic system 
Following the reintroduction of the educational intervention between cycle 3 and 4 (OS figure 2), standards rose again to expectedly increased levels. However the rechecking of pain scores still remained low, further indicating a need for the proposed electronic system, in addition to the aforementioned solutions.

[Online supplement figure 2]

\section{Results}

Interventions and reauditing took place monthly (OS figure 2). Cycle 2 (23 patients) showed significant improvement. $40 \%$ of severe pain scores were now acted upon within recommended timings (OS figure 3 ). $83 \%$ of pain scores were documented at triage, $52 \%$ of prescribed analgesia were guideline compliant, but still only $22 \%$ of pain scores were rechecked within an hour (OS figure 4, table 1).

When no educational intervention was instigated however, results returned to baseline. In cycle 3 (33 patients), zero percent of severe pain scores were acted upon within recommended timings (20 minutes, OS figure 3 ). Though $81 \%$ of pain scores were still documented at triage, there was a drop to $33 \%$ of guideline compliant prescription, and again only $27 \%$ of pain scores were rechecked (OS figure 4). These results reinforce the need for continuous auditing and intervention.

The educational intervention was reimplemented in cycle 4 (30 patients). Forty percent of patients in severe pain were given analgesia within 20 minutes (OS figure 3), 79\% of pain scores were documented at triage, and guideline compliance increased to its maximal level at $57 \%$. The documented rechecking however was again only $20 \%$ (OS figure 4), emphasising the potential importance of an electronic system in not only prompting pain score documentation, but also in rechecking.

See supplementary file: ds6139.pdf - "Online supplement figures 1 $4 "$

\section{Lessons and limitations}

One of the largest barriers to improving standards was a lack of nurse prescriber sign-offs. This meant that even when recognising and documenting pain at triage, analgesia would still be delayed as doctors then need to be sourced to prescribe. The sign-offs would mean nurses could prescribe certain analgesia instantaneously when clinically indicated.

A second limitation was in the study design. If a high pain score e.g. 9/10 was audited with a 'low pain score analgesia' e.g. paracetamol, our audit would have deemed this prescription noncompliant. However, it might be that the patients subjective pain score was documented i.e. the patient's response when asked how severe the pain is on a scale of 1 to 10 ; and that the clinician prescribed analgesia based on his/her objective assessment of pain, being lower. The need for objective pain score documentation was emphasised after cycle 1.
Junior doctors within the emergency department turn over in a four month period hence any intervention in one cohort of doctors would not be reflected as change in the new cohort. The teaching session therefore was integrated into the junior doctor induction programme, with one doctor from each cohort allocated as a pain relief service improvement champion.

Lastly, the set standard the audit was measured against was based on our own departmental adaptation of RCEM guidelines. Though very similar, because of local variance between trusts, these results shouldn't be generalised as a comparison against other departmental improvement initiatives.

\section{Conclusion}

While poor pain management may not impact upon long term patient prognosis it does decrease perception of care. It is the largest cause of attendance to the ED; and additionally with patient satisfaction high on the National Health Service agenda, this justifies an imperative to make sure analgesia is prescribed adequately to all patients in pain.

A multi-faceted approach was taken to maximise adequate prescribing. RCEM guidelines did not include some common and locally used medications; this is likely to be the case in other EDs across the country, being a barrier to guideline use and perpetuating a low awareness amongst junior doctor staff. Integrating local medications as we have would encourage guideline use by increasing its local relevance.

A low incidence of pain score documentation at triage, leading to prescription delays, was the largest factor to missing set standards. A lack of nurse prescriber sign-offs were a significant factor here, and being unable to act upon pain may have fostered apathy to pain score.

Improved standards returned to baseline within a month when no educational intervention was implemented; further, documented rechecking of pain scores remained low throughout the audit. We therefore recommend regular auditing to maintain standards. High intensity workload and cognitive demand are barriers to rechecking, and long term initiatives using technology to streamline the process could be the only way to ensure sustainable change.

\section{References}

1. [cited 2014 28/07]; Available from: http://www.collemergencymed.ac.uk/code/document.asp?ID $=4681$.

2. Tanabe, P. and M. Buschmann, A prospective study of ED pain management practices and the patient's perspective. Journal of Emergency Nursing, 1999. 25(3): p. 171-177.

3. Todd, K.H., et al., Pain in the emergency department: results of the pain and emergency medicine initiative (PEMI) multicenter study. The journal of pain, 2007. 8(6): $p$. 460-466.

4. Guru, V. and I. Dubinsky, The patient vs. caregiver 


\section{BMJ Quality Improvement Reports}

perception of acute pain in the emergency department. The Journal of emergency medicine, 2000. 18(1): p. 7-12.

5. La Vonne, A.D. and L.S. Zun, Pain management in the emergency department and its relationship to patient satisfaction. Journal of Emergencies, Trauma and Shock, 2010. 3(4): p. 326.

6. Taylor, D.M., R. Wolfe, and P.A. Cameron, Complaints from emergency department patients largely result from treatment and communication problems. Emergency Medicine, 2002. 14(1): p. 43-49.

7. Thomas, S.H., Management of pain in the emergency department. ISRN Emergency Medicine, 2013. 2013.

8. Sokoloff, C., et al., Is adequate pain relief and time to analgesia associated with emergency department length of stay? A retrospective study. BMJ open, 2014. 4(3): p. e004288.

9. Baharuddin, K.A., et al., Assessing patient pain scores in the emergency department. The Malaysian journal of medical sciences: MJMS, 2010. 17(1): p. 17.

10. Harper, C., T. Waterfield, and P. Thomas, Multi-disciplinary training improves pain management in the paediatric emergency department. African Journal of Emergency Medicine, 2013. 3(4): p. S5.

\section{Declaration of interests}

No conflict of interests to declare.

\section{Acknowledgements}

We would like to acknowledge the contribution of Thomas McCarthy (project manager - medicine and cardiovascular division, Emergency Department Service Improvement Team [EDSIT] coordinator). Kevin Enright (ED consultant and EDSIT lead), and his successor Sarah Krishnanandan (ED consultant). Jason Fitch (consultant emergency medicine and FY2 teaching co-ordinator) for facilitating the pain relief education sessions, and Lucy Parsons (senior sister) for facilitating the nursing education sessions. Finally Stephen East, whom alongside Sarah Krishnanandan planned the integration of the electronic pain score system.

\section{Ethical approval}

Accepted for audit by the St George's audit department. 